\author{
Martine de Boisdeffre \\ Présidente de la Section du rapport et des études du Conseil d'État
}

\title{
L'ACCÈS AUX DÉCISIONS DES JURIDICTIONS ADMINISTRATIVES ET LEUR PUBLICITÉ
}

\author{
UDK: $342: 347.9$ \\ Primljeno: 1. rujan 2018. \\ Izvorni znanstveni rad
}

Selon Jeremy Bentham : « la publicité est l'âme de la justice ${ }^{1}$. Celle-ci ne saurait être secrète ; elle se doit au contraire d'être transparente. Or cette exigence n'est pleinement satisfaite que lorsque tout citoyen peut avoir connaissance des décisions de justice en y accédant, à la fois matériellement, en les obtenant, et intellectuellement, en en comprenant le sens et la portée. La première dimension renvoie à l'accessibilité stricto sensu et la seconde à l'intelligibilité, pour reprendre la terminologie employée par le Conseil constitutionnel il y a près de vingt ans lorsqu'il a consacré l'objectif à valeur constitutionnelle d'accessibilité et d'intelligibilité du droit ${ }^{2}$.

La justice administrative a engagé une réflexion sur l'accessibilité et l'intelligibilité de ses décisions. Un groupe de travail sur la diffusion de ses productions, présidé par Christian Vigouroux a rendu un rapport en 2010. Un autre groupe de travail, présidé par Philippe Martin a rendu un rapport sur la rédaction des décisions de la juridiction administrative en $2012^{3}$.

Bien que les deux dimensions, matérielle et intellectuelle, de l'accès aux décisions de la justice administrative interagissent, il convient de les distinguer en abordant d'abord l'accessibilité (I) puis l'intelligibilité (II).

Mots clés: decisions, publicité, accessibilité, France

\section{L’ACCESSIBILITÉ DES DÉCISIONS DE LA JUSTICE ADMINISTRATIVE}

L'accessibilité des décisions de la justice administrative dépend de leur diffusion (A), qui peut s'appuyer sur la révolution numérique (B).

\footnotetext{
1 The Works of J. Bentham, éd. John Bowring, W. Tait, Édimbourg, 1843, t. IV, p. 316

2 Décision n ${ }^{\circ}$ 99-421 DC du 16 décembre 1999, Loi portant habilitation du gouvernement à procéder par ordonnances à l'adoption de certains codes

$3 \mathrm{http}: / /$ www. conseil - etat. fr/ Actualites/ Communique s/ Groupe - de - travail-sur-la-redaction-desdecisions-de-la- juridiction-administrative-rapport-final
} 


\section{A. L'accessibilité des décisions de la justice administrative par leur diffusion}

La diffusion des décisions de justice a pour enjeux le contrôle démocratique de cette dernière (1), l'accessibilité du droit (2) et, lorsqu'elle est transfrontière, le rayonnement international de celui-ci (3).

\section{Le contrôle démocratique de la justice}

Le droit d'accès de toute personne aux décisions de justice résulte du principe de leur publicité, énoncé à l'article L.10 du titre préliminaire du code de justice administrative aux termes duquel : «Les jugements sont publics (...)». Il en résulte notamment que les juridictions doivent en principe communiquer leurs décisions à toute personne qui le demande ${ }^{4}$ et ne peuvent exiger en contrepartie que le montant correspondant au coût de la copie ${ }^{5}$.

Cette publicité vise à responsabiliser le juge et à assurer la transparence de la justice $^{6}$. Elle est également une composante du procès équitable garanti par l'article $6 \$ 1$ de la Convention européenne de sauvegarde des droits de l'homme et des libertés fondamentales, aux termes duquel : «Toute personne a droit à ce que sa cause soit entendue (...) publiquement (...). Le jugement doit être rendu publiquement (...)». La Cour européenne des droits de l'homme en a souligné l'importance aux fins de protéger les justiciables contre une justice secrète qui échapperait au contrôle $d u$ public ${ }^{7}$.

\section{L'accessibilité du droit via celle de la jurisprudence}

Rassemblées, les décisions de justice constituent la jurisprudence, dont l'accessibilité est indispensable à celle du droit.

C'est pourquoi le service public de diffusion du droit par internet via le site Légifrance, créé par le décret n ${ }^{\circ} 2002-1064$ du 7 août 2002 présente non seulement les textes mais encore les principales décisions de justice : y sont publiées intégralement les décisions des cours suprêmes - Conseil d'Etat et Cour de cassation - et les décisions les plus importantes des juridictions subordonnées.

${ }^{4}$ CE, $1^{\text {er }}$ décembre 1993, Association SOS Défense et Bertin, $n^{\circ} 95048$

5 Cela résulte de l'applicabilité du régime des archives (articles L.211-1 et L. 211-4 du code du patrimoine relatifs aux archives et aux archives publiques respectivement) dont découle celle de l'article L. 311-9 du code des relations entre le public et l'administration, auquel renvoie l'article L.213-1 du code du patrimoine, et qui dispose que : " L'accès aux documents administratifs s'exerce, au choix du demandeur et dans la limite des possibilités techniques de l'administration : (...) par la délivrance d'une copie (...) aux frais du demandeur, sans que ces frais puissent excéder le coût de cette reproduction (...); $3^{\circ}$ Par courrier électronique et sans frais lorsque le document est disponible sous forme électronique $(\ldots) »$.

6 Voir les conclusions du président Genevois sur l'arrêt de Section du 8 janvier 1982 Serban, n²2729

7 Cour européenne des droits de l'homme, 8 décembre 2003, Pretto et autres contre Italie, nº7984/77, point 21 
De surcroît, la juridiction administrative œuvre, de sa propre initiative, à la diffusion de ses décisions. Conformément à l'une des recommandations du rapport Vigouroux, elle a créé Ariane $w e b^{8}$ : les décisions diffusées sont les mêmes que sur Légifrance, mais avec une valeur ajoutée car elles sont accompagnées des analyses qui mettent en évidence leur apport à la jurisprudence et des conclusions du rapporteur public, si ce dernier a choisi de les diffuser 9 .

La diffusion des décisions de justice, en contribuant à l'accessibilité du droit, est aussi un facteur de rayonnement de ce dernier à l'international.

\section{Le rayonnement du droit français à l'international}

Le Conseil d'Etat promeut une politique de « présence du droit français sur internet », de même que sa traduction, notamment en anglais ${ }^{10}$. S'agissant de ses propres décisions, il rend accessibles les plus importantes sur son site internet en plusieurs langues, via un moteur de recherche. Il est important en effet d'être présent dans le débat juridique mondial, d'offrir des informations en ligne. Car il ne faudrait pas que, comme le déclarait le professeur Gjidara en 2012, «le choix de s'inspirer des solutions anglo-américaines » soit « effectué par défaut, c'est-àdire faute d'accéder aux autres modèles juridiques par manque de connaissances linguistiques $»^{11}$.

\section{B. De nouvelles perspectives de diffusion offertes par la révolution numérique}

Les possibilités techniques de diffusion des décisions de justice, à l'intérieur comme à l'extérieur de nos frontières, sont aujourd'hui décuplées par la transformation numérique, dont une politique volontariste d'ouverture des données peut tirer parti (1) à condition d'en maîtriser les risques (2).

8 Son lancement avait été annoncé par un communiqué de presse du Conseil d'Etat du 12 juillet 2011.

9 Les rapporteurs publics détiennent des droits de propriété intellectuelle sur leurs conclusions qu'ils ne sont par conséquent pas tenus de diffuser, que ce soit spontanément en les publiant ou en permettant leur versement dans des bases de données ou en les communiquant aux justiciables qui en font la demande. Le rapport Vigouroux (p. 21) a jugé important que le rapporteur public demeure « maître de sa décision » quant à la diffusion de ses conclusions, tout en encourageant celle-ci.

10 Conseil d'Etat, L'influence internationale du droit français, étude adoptée par l'Assemblée générale du Conseil d'Etat le 19 juin 2001, La Documentation française, Paris, pp. 106 à 110

${ }_{11}$ M. Gjidara, «L'ouverture de la Croatie aux influences françaises dans l'ordre juridique et institutionnel », Rapport présenté au Colloque international organisé par l'Université Paris Sorbonne et l'Académie croate des sciences et des arts, sur « La Croatie et la France - la Croatie et l'Europe : rapports intellectuels et culturels entre la Croatie et la France à travers l'histoire », les 6 et 7 décembre 2012, à la Sorbonne, pp. 873-874 


\section{Une politique volontariste d'ouverture des données en matière de décisions de justice}

Dès 2010, le rapport Vigouroux constatait que le support papier comme vecteur de la diffusion des décisions de justice était en perte de vitesse et préconisait de privilégier le support numérique ${ }^{12}$. Celui-ci offre d'indéniables avantages techniques, rendant possible la diffusion d'un nombre illimité de décisions à un coût réduit, rapidement et au-delà des frontières.

La loi du 7 octobre 2016 pour une République numérique impose la mise à la disposition du public à titre gratuit de toutes les décisions rendues par les juridictions, administratives ${ }^{13}$ comme judiciaires $^{14}$.

Elle fait écho à la Recommandation du comité des ministres du Conseil de l'Europe $n^{\circ} \mathrm{R}(95) 1$ du 11 septembre 1995 selon laquelle « la pleine connaissance de la jurisprudence de toutes les juridictions est une des conditions essentielles de l'application équitable du droit ». Elle répond aussi à une revendication ancienne des professionnels du droit de pouvoir accéder à l'intégralité des décisions de justice ${ }^{15}$.

\section{La maîtrise des risques de l'ouverture des données en matière de décisions de justice}

L'ouverture des données appliquée aux décisions de justice comporte toutefois un certain nombre de risques. Pour la vie privée des personnes, tout d'abord. A la suite de la recommandation de la CNIL du 29 novembre $2001^{16}$, interprétant la loi du 6 janvier 1978 relative à l'informatique, aux fichiers et aux libertés, les diffuseurs publics de la jurisprudence administrative (Legifrance et Ariane Web) ont procédé à l'anonymisation. Cette approche se justifie par la circonstance que la diffusion publique en ligne accroît considérablement le risque d'atteinte à la vie privée des justiciables ${ }^{17}$.

De ce point de vue, l'approche française, qui repose sur une anonymisation systématique, est exigeante, comme le montre une comparaison internationale : ailleurs, l'anonymisation est réservée à certains contentieux ${ }^{18}$ ou se fait à la demande des justiciables concernés ${ }^{19}$; il n'est généralement pas fait de distinction entre

12 Rapport Vigouroux, p. 31

13 La loi de 2016 a modifié l'article L. 10 du code de justice administrative.

14 La loi de 2016 a créé l'article L. 111-13 du code de l'organisation judiciaire.

15 Rapport Vigouroux, pp. 14-16

16 Délibération $\mathrm{N}^{\circ}$ 01-057 portant recommandation sur la diffusion de données personnelles sur internet par les banques de données de jurisprudence

17 F. Lelièvre, «L'anonymisation à la française des décisions de justice, une exception ? », AJDA 2012, p. 526

18 Ainsi en Belgique, en Italie et au Royaume-Uni : Rapport Vigouroux, p. 11.

19 Ainsi aux Etats-Unis : rapport Vigouroux, p. 11 
support papier et support numérique ${ }^{20}$. Cette approche exigeante ne suffit pourtant pas à assurer une parfaite protection de la vie privée : l'anonymisation telle qu'elle est pratiquée n'empêche pas toute réidentification des personnes ${ }^{21}$.

Elle présente aussi des inconvénients : son coût, mais aussi la perte du moyen mnémotechnique qu'est la désignation d'un arrêt par le nom des parties, traditionnelle en droit administratif français ${ }^{22}$.

En outre, la généralisation de la mise en ligne des décisions de justice risque de donner, à tort, l'impression qu'elles sont toutes de valeur égale, alors que pour comprendre la jurisprudence, «ce qui compte n'est pas l'exhaustivité, mais au contraire la sélection $»^{23}$, le risque étant, pour le public et les justiciables, de se perdre dans « une masse informe et confuse de données $»^{24}$. L'accessibilité accrue des décisions ne doit pas nuire à leur intelligibilité.

Enfin, en ce qui concerne les juges, se pose la question de savoir s'il ne convient pas de supprimer la mention de leurs noms sur les décisions de justice faisant l'objet d'une diffusion massive. La publicité des décisions de justice, pour être complète, impose en principe la mention du nom des juges qui les ont rendues, comme le prévoit l'article L.10 du code de justice administrative ${ }^{25}$. Il en va de même du principe de responsabilité des juges envers le peuple français, au nom duquel ces derniers rendent leurs décisions ${ }^{26}$. Toutefois, la publicité, dès lors qu'elle est pleinement assurée par les modes de diffusion traditionnels des décisions de justice déjà évoqués (voir supra I.A.1.), n'implique pas que le nom des juges soit mentionné sur les décisions de justice diffusées en open data ${ }^{27}$. Une telle mention pourrait décupler les risques pour la sécurité des juges ${ }^{28}$ et ainsi nuire à leur indépendance.

Enfin, mais est-ce un risque, ou une chance, l'ouverture des données en matière de décisions de justice ne manquera pas d'alimenter le marché de la justice dite prédictive, c'est-à-dire de services proposés par des entreprises de legal tech exploitant les données juridiques pour tenter de prédire l'issue d'une action en justice. La mise en évidence des divergences entre les pratiques de différentes juridictions pourrait permettre d'améliorer la prévisibilité de la justice, mais d'un

20 Ainsi en Allemagne, en Espagne, en Italie ou encore au Royaume-Uni et devant la Cour de justice de l'Union européenne et la Cour européenne des droits de l'homme. Voir F. Lelièvre, précité

21 M. Babonneau, T. Coustet, , « Open data des décisions de justice : le casse-tête judiciaire du 21 ème siècle », Dalloz actualité, 10 janvier 2018

22 O. Cachard, " Aux grands arrêts, les juristes reconnaissants... Brefs propos sur 1' "anonymisation" des décisions de justice », D. 2004. 429

23 J.-H. Stahl, «"Open data" et jurisprudence », Droit Administratif, $\mathrm{n}^{\circ}$ 11, Novembre 2016, repère 10

24 Selon l'expression du sénateur Alain Richard, cité par J.-H. Stahl, précité

25 De même d'ailleurs que les articles 454 alinéa 2 et 4 du code de procédure civile et 486 alinéa $1^{\text {er }}$ du code de procédure pénale.

26 Aux termes de l'article L.2 du code de justice administrative comme d'ailleurs de l'article L.111-1 du code de l'organisation judiciaire.

27 Rapport à la ministre de la justice, L'open data des décisions de justice, novembre 2017, dit rapport Cadiet, p. 48

28 Rapport Cadiet, pp. 47-48 
autre côté, « la possibilité d'individualiser certaines tendances juridictionnelles » risque de déboucher sur un forum shopping "visant à contourner le juge naturellement compétent ». Toutefois, la juridiction administrative, du fait de la grande homogénéité de sa jurisprudence, n'aurait pas tellement à craindre le développement de la justice prédictive ${ }^{29}$.

\section{L'INTELLIGIBILITÉ DES DÉCISIONS DE LA JUSTICE ADMINISTRATIVE}

Les décisions de la justice administrative font l'objet, comme toutes les autres, d'une demande plus forte d'intelligibilité de la part de nos concitoyens. Leur rédaction présente en effet des points forts et d'autres qui le sont un peu moins (A). Au-delà de leur hiérarchisation, une évolution de leur rédaction est aujourd'hui à l'œuvre (B).

\section{A. La rédaction des décisions du juge administratif}

La rédaction, c'est d'abord la motivation. Celle des décisions du juge administratif français est conçue comme devant principalement retranscrire le syllogisme juridique, pour assurer la rigueur du raisonnement et ainsi la qualité intrinsèque de la décision ${ }^{30}$. Il en découle un « style apodictique ${ }^{31}$ qui " présente un caractère de nécessité et d'universalité dispensant, par conséquent, d'aller au-delà de l'énoncé de principes lapidaires » s'incarnant dans la phrase unique introduite par un « Considérant $»^{32}$.

Le rapport Martin soulignait son caractère très éloigné du langage courant qui le rend difficile à appréhender par les non initiés. « En choisissant de s'exprimer sur un mode si peu familier au public, la juridiction administrative risque également de nourrir la critique d'une juridiction désuète et isolée de la société $»^{33}$.

Il indiquait aussi que la plupart des juridictions étrangères ont adopté un style plus moderne, abandonnant la phrase unique lorsqu'elles la pratiquaient $t^{34}$. Dans son étude de 2001 déjà citée, le Conseil d'Etat soulignait que « le style apodictique (...)

29 F. Melleray, « La justice administrative doit-elle craindre la « justice prédictive » ?, AJDA 2017

30 Ibidem

31 Décrit par le Conseil d'Etat dans son étude L'influence internationale du droit français précitée, pp. 71,105 et 106 et plus récemment par le vice-président du Conseil d'Etat J.-M. Sauvé dans son discours : « L'influence par le droit », XXIIème Conférence des ambassadeurs, 28 août 2014.

32 J.-M. Sauvé, « L'influence par le droit », précité, note de bas de page n 3

33 Rapport Martin, p. 39

34 Ainsi des juridictions espagnoles, italiennes ou encore du Conseil d'Etat néerlandais : rapport Martin, p. 41. 
Martine de Boisdeffre: L'accès aux décisions des juridictions administratives et leur publicité Zbornik radova Pravnog fakulteta u Splitu, god. 56, 1/2019., str. 47.- 57.

tranche avec ce qui tend à devenir une sorte de standard et ne facilite pas d'emblée, pour un observateur étranger, la compréhension des tenants et aboutissants du dispositif $»^{35}$, recommandant une réforme de la rédaction des décisions de justice en vue de faciliter la comparaison des raisonnements et ainsi le « dialogue des juges ${ }^{36}$.

\section{B. Au-delà de la hiérarchisation des décisions du juge administratif, l'évolution de leur rédaction}

C'est pourquoi, au-delà de la hiérarchisation des décisions du juge administratif français (1), l'évolution de leur rédaction est aujourd'hui à l'œuvre (2).

\section{La tradition de la hiérarchisation des décisions}

Le Conseil d'Etat a toujours effectué un travail de hiérarchisation de ses propres décisions en vue de leur ordonnancement et d'une diffusion sélective, différenciée en fonction de leur intérêt jurisprudentiel : les plus importantes sont publiées au Recueil Lebon, celles présentant un intérêt certain mais moindre sont signalées aux Tables du Recueil Lebon et celles ne présentant pas d'intérêt juridique particulier ne sont pas publiées. On parle de « fichage » des décisions. Il dépend notamment des formations de jugement qui les ont rendues, celles-ci étant elles-mêmes hiérarchisées: pour le Conseil d'Etat, la plus solennelle est l'assemblée ${ }^{37}$ du contentieux, suivie de la section du contentieux ${ }^{38}$, les formations ordinaires étant les chambres ${ }^{39}$, réunies ou jugeant seules ${ }^{40}$.

Le « fichage » précise la portée jurisprudentielle de la solution rendue. C'est en se référant à lui que l'on indique parfois ce qui a été jugé. Il explique comment

35 Conseil d'Etat, L'influence internationale du droit français, précité, p. 71

36 Ibidem

37 Article R. 122-20 du même code : «L'assemblée du contentieux comprend : $1^{\circ}$ Le vice-président du Conseil d'Etat ; $2^{\circ}$ Les présidents de section ; $3^{\circ}$ Les trois présidents adjoints de la section du contentieux ; $4^{\circ}$ Le président de la chambre sur le rapport de laquelle l'affaire est jugée ou, si l'instruction a été faite dans les conditions prévues au premier alinéa de l'article R. 611-20, le président de la chambre à laquelle l'affaire a été initialement attribuée ou, en cas d'examen d'une question de droit renvoyée en application de l'article R. 773-12, le président de la formation spécialisée ; $5^{\circ}$ Les quatre présidents de chambre les plus anciens dans leurs fonctions en dehors du précédent ; $6^{\circ}$ Le rapporteur. La présidence de l'assemblée du contentieux appartient au vice-président du Conseil d'Etat. L'assemblée ne peut valablement siéger que si neuf de ses membres ou leurs suppléants sont présents. L'assemblée du contentieux ne peut statuer qu'en nombre impair. Lorsque les membres présents à la séance ayant voix délibérative sont en nombre pair, l'assemblée est complétée par le président de chambre le plus ancien dans ses fonctions ne siégeant pas au titre du $4^{\circ}$ ou du $5^{\circ}$ ou, à défaut, par l'assesseur le plus ancien dans ses fonctions. »

38 Article R. 122-18 du même code : « La section du contentieux en formation de jugement comprend $: 1^{\circ}$ Le président de la section ; $2^{\circ}$ Les trois présidents adjoints ; $3^{\circ}$ Les présidents de chambre et, en cas d'examen d'une question de droit renvoyée en application de l'article R. 773-12, le président de la formation spécialisée ; $4^{\circ}$ Le rapporteur $»$.

39 L'article 62 de la loi du 20 avril 2016 relative à la déontologie et aux droits et obligations des fonctionnaires a substitué cette dénomination à l'ancienne appellation de "sous-sections ».

40 Article R.122-11 du code de justice administrative 
l'arrêt s'inscrit dans la jurisprudence antérieure. Il explicite également les solutions implicites, par exemple, la compétence de la juridiction administrative ou encore la recevabilité du recours, qui " ne se prêtent pas à la rédaction d'un Considérant de principe puisque le juge n'a pas été saisi de la question » par les parties. Par conséquent, " pour le Conseil d'Etat, autant sinon plus que le texte de la décision juridictionnelle elle-même, hermétique aux non spécialistes, c'est son analyse par la juridiction qu'il convient de diffuser afin de rendre la jurisprudence (...) intelligible $»^{41}$. Cette pratique n'est pas incompatible avec l'accès exhaustif aux décisions de justice ${ }^{42}$, bien au contraire. Elle est aujourd'hui d'autant plus nécessaire que la diffusion des décisions est massive ${ }^{43}$.

\section{Une évolution de la rédaction des décisions du juge administratif est à l'ouvre} Martin.

Elle vise à répondre à différentes préconisations du rapport déjà cité du Président

En premier lieu, « améliorer la lisibilité des décisions de justice » elles-mêmes, en vue de faciliter leur " compréhension par tous les citoyens », autant qu'il est possible, car « compte tenu de la technicité de la matière juridique », " il serait illusoire de viser une parfaite et immédiate compréhension par tous les citoyens de l'ensemble des jugements et arrêts ». Néanmoins, le mode de rédaction ne doit pas constituer « un obstacle supplémentaire et inutile ${ }^{44}$.

En deuxième lieu, assurer l'équilibre entre qualité intrinsèque et intelligibilité de la décision, la première ne devant pas être sacrifiée à la seconde ${ }^{45}$. En effet, si le langage juridique peut évoquer le « jargon » d'une «tribu », c'est d'abord parce qu'il traduit des concepts très affinés. Plutôt que de prendre le risque de les déconstruire ${ }^{46}$, il faut conserver " la richesse de notre langue, reflet de la précision de nos concepts $»^{47}$. Il faut encore tenir compte de contraintes telles que la délibération collégiale des motifs - et non pas seulement du dispositif - au sein de la juridiction administrative française, gage d'une bonne justice, et l'exigence de délais raisonnables de jugement dans un contexte d'accroissement quantitatif du contentieux.

En troisième lieu, enrichir avec modération la motivation. Il s'agit de s'inscrire « dans le respect du principe général selon lequel ne figurent dans la décision que les éléments qui fondent la solution du litige » pour ne pas «brouiller la compréhension

41 P. Cassia, « Une autre manière de dire le droit administratif : le fichage des décisions du Conseil d'Etat au Recueil Lebon », RFDA 2011 p. 830

42 Rapport Vigouroux, p. 17

43 J.-H. Stahl, précité

44 Rapport Martin, p. 11

45 Rapport Martin, 2012

46 B. Sellier «Arrêtons de décoder » AJDA 2005, p. 801

47 D. Chabanol « Tkitoi, Du principe de lisibilité aux SMS », AJDA 2005, p. 1921 
de la décision ». La motivation de demain ne devra donc contenir que ce qui justifie nécessairement la réponse à l'argumentation du justiciable, comme actuellement, mais en l'exprimant le plus clairement et le plus complètement possible ${ }^{48}$. Pour l'enrichir, il conviendra d'expliquer davantage le cadre juridique applicable ou encore les raisons pour lesquelles telle interprétation d'une norme a été retenue ${ }^{49}$.

En quatrième lieu, continuer à citer les décisions de la Cour de justice de l'Union européenne ou encore du Conseil constitutionnel dans les décisions qui les mettent en ouvre ou s'en inspirent. Il est encore recommandé de développer la motivation en fait par des références plus précises aux pièces ayant emporté la conviction du juge et celle de la qualification juridique des faits, qui l'est moins en France qu'à l'étranger ${ }^{50}$. S'agissant du style de la décision, il est proposé d'abandonner les «Considérant » au profit du style direct pour faciliter la lecture et permettre des phrases et paragraphes plus courts ${ }^{51}$.

La mise en œuvre de ces recommandations se fait sur la base de l'expérimentation. Celle-ci a débuté au sein de quatre chambres ${ }^{52}$ de la section du contentieux du Conseil d'Etat ${ }^{53}$; deux autres chambres rédigent aujourd'hui également en style direct, ayant abandonné le «Considérant que » ${ }^{54}$. L'expérimentation a d'abord porté sur les visas, puis sur les motifs ${ }^{55}$. Elle a ensuite été étendue à certaines chambres de certains tribunaux administratifs et de certaines cours administratives d'appel ${ }^{56}$.

La mise en place de l'expérimentation a fait l'objet d'un suivi centralisé, par un comité de pilotage présidé par le président de la section du contentieux qui s'est réuni entre février 2013 et janvier 2015. Aujourd'hui, un Vademecum des nouvelles rédactions est en cours d'élaboration par un groupe de travail présidé par Bernard Stirn, ancien président de la section du contentieux. L'élaboration de modèles est une manière d'encadrer l'évolution des pratiques ; elle avait d'ailleurs été recommandée à ce titre par le rapport Martin ${ }^{57}$. C'est une fois le bilan de l'expérimentation dressé que le processus pourra être étendu à l'ensemble des juridictions ${ }^{58}$.

48 Rapport Martin, p.15

49 Rapport Martin, p. 28

50 Ibidem

51 Rapport Martin, p. 40

52 Qui à l'époque, avant l'adoption de la loi de 2016 précitée, étaient encore des « sous-sections ».

53 Les première, troisième, neuvième et dixième chambres

54 Les sixième et huitième chambres

55 Surtout au sein de la première chambre

56 A compter du printemps 2014 dans sept chambres des cours administratives d'appel de Paris et de Nancy et des tribunaux administratifs de Paris, de Cergy-Pontoise, de Lyon, de Poitiers et de Besançon. D'autres chambres de tribunaux et de cours ont depuis adopté le style direct.

57 Rapport Martin, p. 41

58 Note du chef de la mission permanente d'inspection des juridictions administratives, 5 juillet 2012 


\section{CONCLUSION}

L'amélioration de l'accessibilité et de l'intelligibilité des décisions est l'un des grands chantiers de la juridiction administrative française. Elle rejoint une exigence ancienne de la langue française que rappelait Nicolas Boileau dans L'Art poétique $^{59}$ : «ce que l'on conçoit bien s'énonce clairement. Et les mots pour le dire arrivent aisément ». Elle répond aussi à une demande de transparence caractéristique de notre époque, et au besoin d'accès au droit et à la justice propre à l'Etat de droit et à la démocratie. Mais le plein accès aux décisions de justice suppose aussi la simplification et la qualité du droit, dont les décisions de justice font application. Le Conseil d'Etat y a appelé une nouvelle fois ${ }^{60}$ en 2016 dans son étude annuelle. Il s'agit là d'une entreprise difficile, car la perspective d'un droit simple pour régir une société complexe relève quelque peu de l'utopie ; mais l'utopie peut être utile, dès lors qu'il est possible, si ce n'est d'atteindre l'horizon qu'elle dessine, à tout le moins, de s'en approcher.

\section{PUBLICATION OF AND ACCESS TO ADMINISTRATIVE COURT DECISIONS}

Jeremy Bentham once said: «Publication is the soul of law ». Court decisions cannot and must not only be public, they must be transparent. This demand can be completely fulfilled only when every citizen is able to know about the content of court decisions in such a way that citizens have access to them. Therefore, the citizen must be given intellectual understanding of their meaning and scope. The first dimension is related to access in a narrow or strict sense. The second dimension is related to the understandability of court decisions which implies that when writing court decisions terminology of the Constitutional Council of the Republic of France should be used. This terminology has been used for over twenty odd years and was adopted when the Constitutional Council dedicated itself to the constitutional aim of accessibility and understandability of the law. Even though these two mentioned dimensions, material and intellectual, in approaching administrative court decisions in interaction are, they must be differentiated in a way that enables accessibility and understandability. Improving accessibility and understandability of decisions is one of the main elements towards which French administrative jurisdiction leans. An all-inclusive approach to court decisions also implies bringing in more quality and simpler legal regulations applicable when reaching court decisions. Following all this, analyzed are: accessibility of administrative court decisions, the influence of French administrative law on certain national legislations, the perspective of accessibility taking into account the digital revolution, risk management of accessibility of certain data emerging from court decisions, understandability of administrative court decisions and the formulation thereof. Finally, the need for measures with the aim of developing reaching administrative court decisions is stressed. This includes understanding all citizens, achieving a balance between internal quality and

59 Ed. Denys Thierry, Paris, 1674

60 Après les études de 1991 De la sécurité juridique et de 2006, Sécurité juridique et complexité du droit, La Documentation française, Paris 
Martine de Boisdeffre: L'accès aux décisions des juridictions administratives et leur publicité Zbornik radova Pravnog fakulteta u Splitu, god. 56, 1/2019., str. 47.- 57.

understandability of decisions, all-inclusivity of explaining administrative court decisions and the need to cite European court and Constitutional Council decisions.

Key words: decisions of administrative courts, publications, availability

\section{OBJAVA I PRISTUP ODLUKAMA UPRAVNIH SUDOVA}

Jeremy Bentham je jednom rekao: «Publikacija je duša zakona». Sudske odluke ne mogu i ne moraju biti samo javne, moraju biti transparentne. Taj se zahtjev može u potpunosti ispuniti tek kada svaki građanin može znati o sadržaju sudskih odluka na takav način da građani imaju pristup njima. Dakle, građaninu se mora omogućiti intelektualno razumijevanje njihovog značenja i opsega. Prva dimenzija odnosi se na pristup u uskom ili strogom smislu. Druga dimenzija odnosi se na razumljivost sudskih odluka, što podrazumijeva da se prilikom pisanja sudskih odluka treba koristiti terminologija Ustavnog vijeća Republike Francuske. Ova se terminologija koristi već više od dvadeset godina i usvojena je kada se Ustavno vijeće posvetilo ustavnom cilju dostupnosti i razumljivosti zakona. Te dvije dimenzije, materijalne i intelektualne, moraju biti diferencirane $u$ spoznaji upravno sudskih odluka u interakciji na način koji omogućuje dostupnost i razumljivost. Poboljšanje dostupnosti i razumljivosti odluka jedan je od glavnih elemenata na kojima se naslanja francuska upravna nadležnost. Sveobuhvatni pristup sudskim odlukama također podrazumijeva donošenje kvalitetnijih i jednostavnijih pravnih propisa koji se primjenjuju pri donošenju sudskih odluka. Slijedom svega navedenog, analizirane su: dostupnost upravnih sudskih odluka, utjecaj francuskog upravnog prava na određena nacionalna zakonodavstva, perspektiva pristupačnosti uzimajući u obzir digitalnu revoluciju, upravljanje rizikom dostupnosti određenih podataka koji proizlaze iz sudskih odluka, razumljivost upravnih odluka sudske odluke i njihovu formulaciju. Naposljetku, naglašava se potreba za mjerama s ciljem razvijanja donošenja odluka upravnog suda. To uključuje njihovo razumijevanje od strane građana, postizanje ravnoteže između unutarnje kvalitete i razumljivosti odluka, sveobuhvatnost objašnjavanja odluka upravnih sudova i potrebu navođenja odluka Europskog suda i Ustavnog vijeća.

Ključne riječi: odluke upravnih sudova, publikacija, dostupnost, Francuska 